This postprint is published in:

Applied mathematics and optimization, Volume 78, August 2018, 1-23

DOI: $10.1007 / \mathrm{s} 00245-016-9392-y$

\title{
NONLINEAR ELLIPTIC INCLUSIONS WITH UNILATERAL CONSTRAINT AND DEPENDENCE ON THE GRADIENT
}

\author{
NIKOLAOS S. PAPAGEORGIOU, VICENŢIU D. RĂDULESCU, AND DUŠAN D. REPOVŠ
}

\begin{abstract}
We consider a nonlinear Neumann elliptic inclusion with a source (reaction term) consisting of a convex subdifferential plus a multivalued term depending on the gradient. The convex subdifferential incorporates in our framework problems with unilateral constraints (variational inequalities). Using topological methods and the Moreau-Yosida approximations of the subdifferential term, we establish the existence of a smooth solution.
\end{abstract}

\section{INTRODUCTION}

Let $\Omega \subseteq \mathbb{R}^{N}$ be a bounded domain with a $C^{2}$-boundary $\partial \Omega$. In this paper we study the following nonlinear Neumann elliptic differential inclusion

$$
\left\{\begin{array}{ll}
\operatorname{div}(a(u(z)) D u(z)) \in \partial \varphi(u(z))+F(z, u(z), D u(z)) & \text { in } \Omega, \\
\frac{\partial u}{\partial n}=0 & \text { on } \partial \Omega .
\end{array}\right\}
$$

In this problem, $\varphi \in \Gamma_{0}(\mathbb{R})$ (that is, $\varphi: \mathbb{R} \rightarrow \overline{\mathbb{R}}=\mathbb{R} \cup\{+\infty\}$ is proper, convex and lower semicontinuous, see Section 2) and $\partial \varphi(x)$ is the subdifferential of $\varphi(\cdot)$ in the sense of convex analysis. Also $F(z, x, \xi)$ is a multivalued term with closed convex values depending on the gradient of $u$. So, problem (1) incorporates variational inequalities with a multivalued reaction term.

By a solution of problem (1), we understand a function $u \in H^{1}(\Omega)$ such that we can find $g, f \in L^{2}(\Omega)$ for which we have

$$
\begin{aligned}
& g(z) \in \partial \varphi(u(z)) \text { and } f(z) \in F(z, u(z), D u(z)) \text { for almost all } z \in \Omega, \\
& \int_{\Omega} a(u(z))(D u, D h)_{\mathbb{R}^{N}} d z+\int_{\Omega}(g(z)+f(z)) h(z) d z=0 \text { for all } h \in H^{1}(\Omega) .
\end{aligned}
$$

The presence of the gradient in the multifunction $F$, precludes the use of variational methods in the analysis of (1). To deal with such problems, a variety of methods have been proposed. Indicatively, we mention the works of Amann and Crandall [1], de Figueiredo, Girardi and Matzeu [5], Girardi and Matzeu [8], Loc and Schmitt [13], Pohozaev [20]. All these papers consider problems with no unilateral constraint (that is, $\varphi=0$ ) and the reaction term $F$ is single-valued. Variational inequalities (that is, problems where $\varphi$ is the indicator function of a closed, convex set), were investigated by Arcoya, Carmona and Martinez Aparicio [2], Matzeu and Servadei [15], Mokrane and Murat [17]. All have single valued source term.

Our method of proof is topological and it is based on a slight variant of Theorem 8 of Bader [3] (a multivalued alternative theorem). Also, our method uses

Key words and phrases. Convex subdifferential, Moreau-Yosida approximation, elliptic differential inclusion, Morse iteration technique, pseudomonotone map, variational inequality.

2010 AMS Subject Classification: 35J60, 35K85. 
approximations of $\varphi$ and the theory of nonlinear operators of monotone type. In the next section, we recall the basic notions and mathematical tools which we will use in the sequel.

\section{Mathematical Background}

Let $X$ be a Banach space and $X^{*}$ be its topological dual. By $\langle\cdot, \cdot\rangle$ we denote the duality brackets for the pair $\left(X^{*}, X\right)$. By $\Gamma_{0}(X)$ we denote the cone of all convex functions $\varphi: X \rightarrow \overline{\mathbb{R}}=\mathbb{R} \cup\{+\infty\}$ which are proper (that is, not identically $+\infty$ ) and lower semicontinuous. By $\operatorname{dom} \varphi$ we denote the effective domain of $\varphi$, that is,

$$
\operatorname{dom} \varphi:=\{u \in X: \varphi(u)<+\infty\} .
$$

Given $\varphi \in \Gamma_{0}(X)$, the subdifferential of $\varphi$ at $u \in X$ is the set

$$
\partial \varphi(u)=\left\{u^{*} \in X^{*}:\left\langle u^{*}, h\right\rangle \leqslant \varphi(u+h)-\varphi(u) \text { for all } h \in X\right\} .
$$

Evidently $\partial \varphi(u) \subseteq X^{*}$ is $w^{*}$-closed, convex and possibly empty. If $\varphi$ is continuous at $u \in X$, then $\partial \varphi(u) \subseteq X^{*}$ is nonempty, $w^{*}$-compact and convex. Moreover, if $\varphi$ is Gâteaux differentiable at $u \in X$, then $\partial \varphi(u)=\left\{\varphi_{G}^{\prime}(u)\right\}\left(\varphi_{G}^{\prime}(u)\right.$ being the Gâteaux derivative of $\varphi$ at $u$ ). We know that the map $\partial \varphi: X \rightarrow 2^{X^{*}}$ is maximal monotone. If $X=H=$ a Hilbert space and $\varphi \in \Gamma_{0}(H)$, then for every $\lambda>0$, the "Moreau-Yosida approximation" $\varphi_{\lambda}$ of $\varphi$, is defined by

$$
\varphi_{\lambda}(u)=\inf \left[\varphi(h)+\frac{1}{2 \lambda}\|h-u\|^{2}: h \in H\right] \text { for all } u \in H .
$$

We have the following properties:

- $\varphi_{\lambda}$ is convex, dom $\varphi_{\lambda}=H$;

- $\varphi_{\lambda}$ is Fréchet differentiable and the Fréchet derivative $\varphi_{\lambda}^{\prime}$ is Lipschitz continuous with Lipschitz constant $1 / \lambda$;

- if $\lambda_{n} \rightarrow 0, u_{n} \rightarrow u$ in $H, \varphi_{\lambda_{n}}^{\prime}\left(u_{n}\right) \stackrel{w^{*}}{\rightarrow} u^{*}$ in $H$, then $u^{*} \in \partial \varphi(u)$.

We refer for details to Gasinski and Papageorgiou [6] and Papageorgiou and Kyritsi [19].

We know that if $\varphi \in \Gamma_{0}(X)$, then $\varphi$ is locally Lipschitz in the interior of its effective domain (that is, on $\operatorname{int} \operatorname{dom} \varphi$ ). So, locally Lipschitz functions are the natural candidate to extend the subdifferential theory of convex functions.

We say that $\varphi: X \rightarrow \mathbb{R}$ is locally Lipschitz if for every $u \in X$ we can find $U$ a neighborhood of $u$ and a constant $k>0$ such that

$$
|\varphi(v)-\varphi(y)| \leqslant k\|v-y\| \text { for all } v, y \in U .
$$

For such functions we can define the generalized directional derivative $\varphi^{0}(u ; h)$ by

$$
\varphi^{\circ}(u ; h)=\limsup _{\substack{u^{\prime} \rightarrow u \\ \lambda \downarrow 0}} \frac{\varphi\left(u^{\prime}+\lambda h\right)-\varphi\left(u^{\prime}\right)}{\lambda} .
$$

Then $\varphi^{\circ}(u ; \cdot)$ is sublinear continuous and so we can define the nonempty $w^{*}$ compact set $\partial_{c} \varphi(u)$ by

$$
\partial_{c} \varphi(u)=\left\{u^{*} \in X^{*}:\left\langle u^{*}, h\right\rangle \leqslant \varphi^{\circ}(u ; h) \text { for all } h \in X\right\} .
$$

We say that $\partial_{c} \varphi(u)$ is the "Clarke subdifferential" of $\varphi$ at $u \in X$. In contrast to the convex subdifferential, the Clarke subdifferential is always nonempty. Moreover, 
if $\varphi$ is convex, continuous (hence locally Lipschitz on $X$ ), then the two subdifferentials coincide, that is, $\partial \varphi(u)=\partial_{c} \varphi(u)$ for all $u \in X$. For further details we refer to Clarke [4].

Suppose that $X$ is a reflexive Banach space and $A: X \rightarrow X^{*}$ a map. We say that $A$ is "pseudomonotone", if the following two conditions hold:

- $A$ is continuous from every finite dimensional subspace $V$ of $X$ into $X^{*}$ furnished with the weak topology;

- if $u_{n} \stackrel{w}{\rightarrow} u$ in $X, A\left(u_{n}\right) \stackrel{w}{\rightarrow} u^{*}$ in $X^{*}$ and $\limsup _{n \rightarrow \infty}\left\langle A\left(u_{n}\right), u_{n}-u\right\rangle \leqslant 0$, then for every $y \in X$, we have

$$
\langle A(u), u-y\rangle \leqslant \liminf _{n \rightarrow \infty}\left\langle A\left(u_{n}\right), u_{n}-y\right\rangle .
$$

If $A: X \rightarrow X^{*}$ is maximal monotone, then $A$ is pseudomonotone.

A pseudomonotone map $A: X \rightarrow X^{*}$ which is strongly coercive, that is,

$$
\frac{\langle A(u), u\rangle}{\|u\|} \rightarrow+\infty \text { as }\|u\| \rightarrow \infty
$$

it is surjective (see Gasinski and Papageorgiou [6, p. 336]).

Let $V$ be a set and let $G: V \rightarrow 2^{X^{*}} \backslash\{\emptyset\}$ be a multifunction. The graph of $G$ is the set

$$
\operatorname{Gr} G=\{(v, u) \in V \times X: u \in G(v)\} .
$$

(a) If $V$ is a Hausdorff topological space and $\operatorname{Gr} G \subseteq V \times X$ is closed, then we say that $G$ is "closed".

(b) If there is a $\sigma$-field $\Sigma$ defined on $V$ and $\operatorname{Gr} G \subseteq \Sigma \times B(X)$, with $B(X)$ being the Borel $\sigma$-field of $X$, then we say that $G$ is "graph measurable".

As we already mentioned in the Introduction, our approach uses a slight variant of Theorem 8 of Bader [3] in which the Banach space $V$ is replaced by its dual $V^{*}$ equipped with the $w^{*}$-topology. A careful reading of the proof of Bader [3], reveals that the result remains true if we make this change.

So, as above $X$ is a Banach space, $V^{*}$ is a dual Banach space, $G: X \rightarrow 2^{V^{*}}$ is a multifunction with nonempty, $w^{*}$-compact, convex values. We assume that $G(\cdot)$ is "upper semicontinuous" (usc for short), from $X$ with the norm topology into $V^{*}$ with the $w^{*}$-topology (denoted by $V_{w^{*}}^{*}$ ), that is, for all $U \subseteq V^{*} w^{*}$-open, we have

$$
G^{-}(U)=\{x \in X: G(x) \cap U \neq \emptyset\} \text { is open. }
$$

Note that if $\operatorname{Gr} G \subseteq X \times V_{w^{*}}^{*}$ is closed and $G(\cdot)$ is locally compact into $V_{w^{*}}^{*}$, that is, for all $u \in X$ we can find $U$ a neighborhood of $u$ such that $\overline{G(U)} w^{w^{*}}$ is $w^{*}$-compact in $V^{*}$, then $G$ is usc from $X$ into $V_{w^{*}}^{*}$. Also, let $K: V_{w^{*}}^{*} \rightarrow X$ be a sequentially continuous map. Then the nonlinear alternative theorem of Bader [3], reads as follows.

Theorem 1. Assume that $G$ and $K$ are as above and $S=K \circ G: X \rightarrow 2^{X} \backslash\{\emptyset\}$ maps bounded sets into relatively compact sets. Define

$$
E=\{u \in X: u \in t S(u) \text { for some } t \in(0,1)\} .
$$

Then either $E$ is unbounded or $S(\cdot)$ admits a fixed point. 


\section{Existence Theorem}

In this section we prove an existence theorem for problem (1). We start by introducing the hypotheses on the data of problem (1).

$H(a): a: \mathbb{R} \rightarrow \mathbb{R}$ is a function which satisfies

$$
\begin{aligned}
& |a(x)-a(y)| \leqslant k|x-y| \text { for all } x, y \in \mathbb{R}, \text { some } k>0, \\
& 0<c_{1} \leqslant a(x) \leqslant c_{2} \text { for all } x \in \mathbb{R} .
\end{aligned}
$$

$H(\varphi): \varphi \in \Gamma_{0}(\mathbb{R})$ and $0 \in \partial \varphi(0)$.

Remark 1. We recall that in $\mathbb{R} \times \mathbb{R}$, every maximal monotone set is of the subdifferential type. In higher dimensions this is no longer true (see Papageorgiou and Kyritsi [19, p. 175]).

$H(F): F: \Omega \times \mathbb{R} \times \mathbb{R}^{N} \rightarrow P_{f_{c}}(\mathbb{R})$ is a multifunction such that

(i) for all $(x, \xi) \in \mathbb{R} \times \mathbb{R}^{N}, z \mapsto F(z, x, \xi)$ is graph measurable;

(ii) for almost all $z \in \Omega,(x, \xi) \mapsto F(z, x, \xi)$ is closed;

(iii) for almost all $z \in \Omega$ and all $(x, \xi, v) \in \operatorname{Gr} F(z, \cdot, \cdot)$, we have

$$
|v| \leqslant \gamma_{1}(z,|x|)+\gamma_{2}(z,|x|)|\xi|
$$

with

$$
\begin{aligned}
& \sup \left[\gamma_{1}(z, s): 0 \leqslant s \leqslant k\right] \leqslant \eta_{1, k}(z) \text { for almost all } z \in \Omega, \\
& \sup \left[\gamma_{2}(z, s): 0 \leqslant s \leqslant k\right] \leqslant \eta_{2, k}(z) \text { for almost all } z \in \Omega,
\end{aligned}
$$

and $\quad \eta_{1, k}, \eta_{2, k} \in L^{\infty}(\Omega)$;

(iv) there exists $M>0$ such that if $\left|x_{0}\right|>M$, then we can find $\delta>0$ and $\eta>0$ such that

$\inf \left[v x+c_{1}|\xi|^{2}:\left|x-x_{0}\right|+|\xi| \leqslant \delta, v \in F(z, x, \xi)\right] \geqslant \eta>0$ for almost all $z \in \Omega$, with $c_{1}>0$ as in hypothesis $H(a)$;

(v) for almost all $z \in \Omega$ and all $(x, \xi, v) \in \operatorname{Gr} F(z, \cdot, \cdot)$, we have

$$
v x \geqslant-c_{3}|x|^{2}-c_{4}|x| \xi\left|-\gamma_{3}(z)\right| x \mid
$$

with $c_{3}, c_{4}>0$ and $\gamma_{3} \in L^{1}(\Omega)_{+}$.

Remark 2. Hypothesis $H(F)(i v)$ is an extension to multifunctions of the NagumoHartman condition for continuous vector fields (see Hartman [9, p. 433], Knobloch [11] and Mawhin [16]).

Let $\hat{a}: H^{1}(\Omega) \rightarrow H^{1}(\Omega)^{*}$ be the nonlinear continuous map defined by

$$
\langle\hat{a}(u), h\rangle=\int_{\Omega} a(u)(D u, D h)_{\mathbb{R}^{N}} d z \text { for all } u, h \in H^{1}(\Omega) .
$$

Proposition 2. If hypotheses $H(a)$ hold, then the map $\hat{a}: H^{1}(\Omega) \rightarrow H^{1}(\Omega)^{*}$ defined by (2) is pseudomonotone.

Proof. Evidently $\hat{a}(\cdot)$ is bounded (that is, maps bounded sets to bounded sets), see hypotheses $H(a)$ and it is defined on all of $H^{1}(\Omega)$. So, in order to prove the desired pseudomonotonicity of $\hat{a}(\cdot)$, it suffices to show the following:

$(G P):$ "If $u_{n} \stackrel{w}{\rightarrow} u$ in $H^{1}(\Omega), \hat{a}\left(u_{n}\right) \stackrel{w}{\rightarrow} u^{*}$ in $H^{1}(\Omega)^{*}$ and $\limsup _{n \rightarrow \infty}\left\langle\hat{a}\left(u_{n}\right), u_{n}-u\right\rangle \leqslant$ 0 , 
then $u^{*}=\hat{a}(u)$ and $\left\langle\hat{a}\left(u_{n}\right), u_{n}\right\rangle \rightarrow\langle\hat{a}(u), u\rangle "$

(see Gasinski and Papageorgiou [6], Proposition 3.2.49, p. 333).

So, according to (GP) above we consider a sequence $\left\{u_{n}\right\}_{n \geqslant 1} \subseteq H^{1}(\Omega)$ such that

$$
u_{n} \stackrel{w}{\rightarrow} u \text { in } H^{1}(\Omega), \hat{a}\left(u_{n}\right) \stackrel{w}{\rightarrow} u^{*} \text { in } H^{1}(\Omega)^{*} \text { and } \limsup _{n \rightarrow \infty}\left\langle\hat{a}\left(u_{n}\right), u_{n}-u\right\rangle \leqslant 0 .
$$

We have

$$
\begin{aligned}
\left\langle\hat{a}\left(u_{n}\right), u_{n}-u\right\rangle & =\int_{\Omega} a\left(u_{n}\right)\left(D u_{n}, D u_{n}-D u\right)_{\mathbb{R}^{N}} d z \\
& =\int_{\Omega} a\left(u_{n}\right)\left|D u_{n}-D u\right|^{2} d z+\int_{\Omega} a\left(u_{n}\right)\left(D u, D u_{n}-D u\right)_{\mathbb{R}^{N}} d z .
\end{aligned}
$$

Hypotheses $H(a)$ and (3) imply that

$$
\int_{\Omega} a\left(u_{n}\right)\left(D u, D u_{n}-D u\right)_{\mathbb{R}^{N}} d z \rightarrow 0 \text { as } n \rightarrow \infty .
$$

Also we have

$$
\begin{aligned}
& \left.\int_{\Omega} a\left(u_{n}\right)\left|D u_{n}-D u\right|^{2} d z \geqslant c_{1}\left\|D u_{n}-D u\right\|_{2}^{2} \text { (see hypotheses } H(a)\right), \\
\Rightarrow & D u_{n} \rightarrow D u \text { in } L^{2}\left(\Omega, \mathbb{R}^{N}\right)(\text { see }(3),(4),(5)) \\
(6) \Rightarrow & u_{n} \rightarrow u \text { in } H^{1}(\Omega)(\operatorname{see}(3)) .
\end{aligned}
$$

For all $h \in H^{1}(\Omega)$, we have

$$
\begin{aligned}
& \left\langle\hat{a}\left(u_{n}\right), h\right\rangle=\int_{\Omega} a\left(u_{n}\right)\left(D u_{n}, D h\right)_{\mathbb{R}^{N}} d z \rightarrow \int_{\Omega} a(u)(D u, D h)_{\mathbb{R}^{N}} d z=\langle\hat{a}(u), h\rangle \\
& \quad \\
\Rightarrow & \hat{a}\left(u_{n}\right) \stackrel{w}{\rightarrow} \hat{a}(u) \text { in } H^{1}(\Omega)^{*}, \\
\Rightarrow & \hat{a}(u)=u^{*}(\text { see }(3)) .
\end{aligned}
$$

From (6) and the continuity of $a(\cdot)$ (see hypotheses $H(a)$ ), we have

$$
\left\langle\hat{a}\left(u_{n}\right), u_{n}\right\rangle \rightarrow\langle\hat{a}(u), u\rangle .
$$

Therefore property (GP) is satisfied and so we conclude that $\hat{a}(\cdot)$ is pseudomonotone.

Next we will approximate problem (1) using the Moreau-Yosida approximations of $\varphi \in \Gamma_{0}(\mathbb{R})$. For this approach to lead to a solution of problem (1), we need to have a priori bounds for the approximate solutions. The proposition which follows is a crucial step in this direction. Its proof is based on the so-called "Morse iteration technique".

So, we consider the following nonlinear Neumann problem:

$$
\left\{\begin{array}{ll}
-\operatorname{div}(a(u(z)) D u(z))=g(z, u(z)) & \text { in } \Omega, \\
\frac{\partial u}{\partial n}=0 & \text { on } \partial \Omega .
\end{array}\right\}
$$

The conditions on the reaction term $g(z, x)$ are the following:

$H(g): g: \Omega \times \mathbb{R} \rightarrow \mathbb{R}$ is a Carathéodory function (that is, for all $x \in \mathbb{R}$, $z \mapsto g(z, x)$ is measurable and for almost all $z \in \Omega, x \mapsto g(z, x)$ is continuous) and

$$
|g(z, x)| \leqslant a(z)\left(1+|x|^{r-1}\right) \text { for almost all } z \in \Omega \text {, all } x \in \mathbb{R},
$$


with $a \in L^{\infty}(\Omega)_{+}, 2 \leqslant r<2^{*}=\left\{\begin{array}{ll}\frac{2 N}{N-2} & \text { if } N \geqslant 3 \\ +\infty & \text { if } N=1,2\end{array}\right.$ (the critical Sobolev exponent).

By a weak solution of problem (7), we understand a function $u \in H^{1}(\Omega)$ such that

$$
\int_{\Omega} a(u)(D u, D h)_{\mathbb{R}^{N}} d z=\int_{\Omega} g(z, u) h d z \text { for all } h \in H^{1}(\Omega) .
$$

Proposition 3. If hypothesis $H(g)$ holds and $u \in H^{1}(\Omega)$ is a nontrivial weak solution of (7), then $u \in L^{\infty}(\Omega)$ and $\|u\|_{\infty} \leqslant M=M\left(\|a\|_{\infty}, N, 2,\|u\|_{2^{*}}\right)$.

Proof. Let $p_{0}=2^{*}$ and $p_{n+1}=2^{*}+\frac{2^{*}}{2}\left(p_{n}-r\right)$ for all $n \in \mathbb{N}_{0}$. Evidently $\left\{p_{n}\right\}_{n \geqslant 0}$ is increasing. First suppose that $u \geqslant 0$. For every $k \in \mathbb{N}$ we set

$$
u_{k}=\min \{u, k\} \in H^{1}(\Omega) .
$$

Let $\vartheta=p_{n}-r>0$ (note that $\left.p_{n} \geqslant 2^{*}>r\right)$. We have

$$
\hat{a}(u)=N_{g}(u) \text { in } H^{1}(\Omega)^{*}
$$

with $N_{g}(u)(\cdot)=g(\cdot, u(\cdot)) \in L^{r^{\prime}}(\Omega) \subseteq H^{1}(\Omega)^{*}, \frac{1}{r}+\frac{1}{r^{\prime}}=1$ (the Nemytskii map corresponding to $g$ ). On (9) we act with $u_{k}^{\vartheta+1}$ (see (8)). Then

$$
\left\langle\hat{a}(u), u_{k}^{\vartheta+1}\right\rangle=\int_{\Omega} g(z, u) u_{k}^{\vartheta+1} d z
$$

Note that

$$
\begin{aligned}
& \left\langle\hat{a}(u), u_{k}^{\vartheta+1}\right\rangle=\int_{\Omega} a(u)\left(D u, D u_{k}^{\vartheta+1}\right)_{\mathbb{R}^{N}} d z \\
= & (\vartheta+1) \int_{\Omega} u_{k}^{\vartheta} a(u)\left(D u, D u_{k}\right)_{\mathbb{R}^{N}} d z \\
\geqslant & \left.(\vartheta+1) \int_{\Omega} u_{k}^{\vartheta} c_{1}\left|D u_{k}\right|^{2} d z \text { (see hypothesis } \mathrm{H}(\mathrm{a}) \text { and recall that } u \geqslant 0\right) \\
(11)= & c_{1}(\vartheta+1) \frac{2}{\vartheta+2} \int_{\Omega}\left|D u_{k}^{\frac{\vartheta+2}{2}}\right|^{2} d z .
\end{aligned}
$$

Also we have

$$
\begin{aligned}
& \int_{\Omega} g(z, u) u_{k}^{\vartheta+1} d z \\
& \leqslant \int_{\Omega} a(z)\left(1+u^{r-1}\right) u^{\vartheta+1} d z(\text { see hypothesis } H(g),(8) \text { and recall } u \geqslant 0) \\
&(12) \leqslant c_{3}\left(1+\int_{\Omega} u^{p_{n}} d z\right) \text { for some } c_{3}>0\left(\text { since } \vartheta+1<\vartheta+r=p_{n}\right) .
\end{aligned}
$$

We return to (10) and use (11) and (12). Then

$$
\begin{aligned}
& c_{1}(\vartheta+1) \frac{2}{\vartheta+2} \int_{\Omega}\left[\left|D u_{k}^{\frac{\vartheta+2}{2}}\right|^{2}+\left|u_{k}^{\frac{\vartheta+2}{2}}\right|^{2}\right] d z \\
\leqslant & c_{4}\left(1+\int_{\Omega} u^{p_{n}} d z\right) \text { for some } c_{4}>0\left(\text { since } \vartheta+r=p_{n}\right) \\
\Rightarrow \quad & \left\|u_{k}^{\frac{\vartheta+2}{2}}\right\|^{2} \leqslant c_{5}\left(1+\int_{\Omega} u^{p_{n}} d z\right) \text { for some } c_{5}>0, \text { all } k \in \mathbb{N}, \text { and } n \in \mathbb{N}_{0} .
\end{aligned}
$$


Here $\|\cdot\|$ denotes the norm of $H^{1}(\Omega)$ (recall that $\|v\|=\left[\|v\|_{2}^{2}+\|D v\|_{2}^{2}\right]^{1 / 2}$ for all $\left.v \in H^{1}(\Omega)\right)$.

By the Sobolev embedding theorem (see (8) and note that $H^{1}(\Omega) \hookrightarrow L^{\frac{{ }^{2} p_{n+1}}{p_{n}}}(\Omega)$ ) we have

$$
\left\|u_{k}\right\|_{p_{n+1}}^{p_{n}} \leqslant c_{6}\left(1+\int_{\Omega} u^{p_{n}} d z\right) \text { for some } c_{6}>0 \text {, all } k \in \mathbb{N}_{0} \text { and } n \in \mathbb{N} \text {. }
$$

Let $k \rightarrow \infty$. Then $u_{k}(z) \uparrow u(z)$ for almost all $z \in \Omega$ (see (8)). So, by the monotone convergence theorem, we have

$$
\left(\int_{\Omega} u^{p_{n+1}} d z\right)^{\frac{p_{n}}{p_{n+1}}} \leqslant c_{6}\left(1+\int_{\Omega} u^{p_{n}} d z\right) \text { for all } n \in \mathbb{N}_{0} .
$$

Recall that $p_{0}=2^{*}$ and by the Sobolev embedding theorem we have $u \in L^{2^{*}}(\Omega)$. So, from (13) and by induction we infer that $u \in L^{p_{n}}(\Omega)$ for all $n \in \mathbb{N}_{0}$. Also we have

$$
\|u\|_{p_{n+1}}^{p_{n}} \leqslant c_{6}\left(1+\|u\|_{p_{n}}^{p_{n}}\right) \text { for all } n \in \mathbb{N}_{0}(\text { see }(13))
$$

Since $p_{n}<p_{n+1}$, using the Hölder and Young inequalities (the latter with $\epsilon>0$ small), we obtain

$$
\|u\|_{p_{n}} \leqslant c_{7} \text { for some } c_{7}>0, \text { all } n \in \mathbb{N}_{0} .
$$

Claim 1. $p_{n} \rightarrow \infty$.

Arguing by contradiction, suppose that the claim were not true. Since $\left\{p_{n}\right\}_{n \in \mathbb{N}_{0}}$ is increasing, we have

$$
p_{n} \rightarrow p_{*}>2^{*}
$$

By definition

$$
\begin{aligned}
& p_{n+1}=2^{*}+\frac{2^{*}}{2}\left(p_{n}-r\right), \\
\Rightarrow & p_{*}=2^{*}+\frac{2^{*}}{2}\left(p_{*}-r\right)(\text { see }(15)) \\
\Rightarrow & p_{*}\left(\frac{2^{*}}{2}-1\right)=2^{*}\left(\frac{r}{2}-1\right)<2^{*}\left(\frac{2^{*}}{2}-1\right)\left(\text { since } 2 \leqslant r<2^{*}\right), \\
\Rightarrow & \left.p_{*}<2^{*}, \text { a contradiction (see } 15\right) .
\end{aligned}
$$

This proves Claim 1.

So, passing to the limit as $n \rightarrow \infty$ in (14), it follows from Gasinski and Papageorgiou [7, p. 477] that

$$
\|u\|_{\infty} \leqslant c_{7}, \text { hence } u \in L^{\infty}(\Omega) .
$$

Moreover, it is clear from the above proof that $\|u\|_{\infty} \leqslant M=M\left(\|a\|_{\infty}, N, 2,\|u\|_{2^{*}}\right)$.

Finally for the general case, we write $u=u^{+}-u^{-}$, with $u^{ \pm}=\max \{ \pm u, 0\} \geqslant 0$ and work with each one separately as above, to conclude $u^{ \pm} \in L^{\infty}(\Omega)$, hence $u \in L^{\infty}(\Omega)$.

Now for $\lambda>0$, let $\varphi_{\lambda}$ be the Moreau-Yosida approximation of $\varphi \in \Gamma_{0}(\mathbb{R})$ and for $\vartheta \in L^{\infty}(\Omega)$, consider the following auxiliary Neumann problem:

$$
\left\{\begin{array}{ll}
-\operatorname{div}(a(u(z)) D u(z))+u(z)+\varphi_{\lambda}^{\prime}(u(z))=\vartheta(z) & \text { in } \Omega, \\
\frac{\partial u}{\partial n}=0 & \text { on } \partial \Omega
\end{array}\right\}
$$


Proposition 4. If hypotheses $H(a), H(\varphi)$ hold and $\vartheta \in L^{\infty}(\Omega)$, then problem (16) admits a unique solution $u \in C^{1}(\bar{\Omega})$.

Proof. Let $V_{\lambda}: H^{1}(\Omega) \rightarrow H^{1}(\Omega)^{*}$ be the nonlinear map defined by

$$
V_{\lambda}(u)=\hat{a}(u)+u+N_{\varphi_{\lambda}^{\prime}}(u) \text { for all } u \in H^{1}(\Omega) .
$$

As before $N_{\varphi_{\lambda}^{\prime}}(u)$ is the Nemytskii map corresponding to $\varphi_{\lambda}^{\prime}$ (that is, $N_{\varphi_{\lambda}^{\prime}}(u)(\cdot)=$ $\left.\varphi_{\lambda}^{\prime}(u(\cdot))\right)$. We have

$$
\begin{aligned}
\left\langle V_{\lambda}(u), u\right\rangle= & \langle\hat{a}(u), u\rangle+\|u\|_{2}^{2}+\int_{\Omega} \varphi_{\lambda}^{\prime}(u) u d z \\
\geqslant & c_{1}\|D u\|_{2}^{2}+\|u\|_{2}^{2} \\
& \left(\text { see hypothesis } H(a) \text { and recall that } \varphi_{\lambda}^{\prime} \text { is increasing, } \varphi_{\lambda}^{\prime}(0)=0\right), \\
(17) \Rightarrow \quad & V_{\lambda} \text { is strongly coercive. }
\end{aligned}
$$

Using the Sobolev embedding theorem we see that $u \mapsto N_{\varphi_{\lambda}^{\prime}}(u)$ is completely continuous from $H^{1}(\Omega)$ into $H^{1}(\Omega)^{*}$ (that is, if $u_{n} \stackrel{w}{\rightarrow} u$ in $H^{1}(\Omega)$, then $N_{\varphi_{\lambda}^{\prime}}\left(u_{n}\right) \rightarrow$ $N_{\varphi_{\lambda}^{\prime}}(u)$ in $\left.H^{1}(\Omega)^{*}\right)$, hence it is pseudomonotone. From Proposition 2 we know that $\hat{a}(\cdot)$ is pseudomonotone and of course the same is true for the embedding $H^{1}(\Omega) \hookrightarrow H^{1}(\Omega)^{*}$ (which is compact). So, from Gasinski and Papageorgiou [6], Proposition 3.2.51, p. 334, we infer that

$$
u \mapsto V_{\lambda}(u) \text { is pseudomonotone. }
$$

Recall that a pseudomonotone strongly coercive map is surjective. So, from (17), (18) it follows that there exists $u \in H^{1}(\Omega)$ such that

$$
\begin{aligned}
& V_{\lambda}(u)=\vartheta \\
& \int_{\Omega} a(u)(D u, D h)_{\mathbb{R}^{N}} d z+\int_{\Omega} u h d z+\int_{\Omega} \varphi_{\lambda}^{\prime}(u) h d z=\int_{\Omega} \vartheta h d z \text { for all } h \in H^{1}(\Omega) .
\end{aligned}
$$

From the nonlinear Green's identity (see Gasinski and Papageorgiou [6], Theorem 2.4 .53 , p. 210), we have

$$
\int_{\Omega} a(u)(D u, D h)_{\mathbb{R}^{N}} d z=\langle-\operatorname{div}(a(u) D u), h\rangle+\left\langle a(u) \frac{\partial u}{\partial n}, h\right\rangle_{\partial \Omega} \text { for all } h \in H^{1}(\Omega),
$$

where by $\langle\cdot, \cdot\rangle_{\partial \Omega}$ we denote the duality brackets for the pair $\left(H^{-\frac{1}{2}, 2}(\partial \Omega), H^{\frac{1}{2}, 2}(\partial \Omega)\right)$.

From the representation theorem for the elements of $H^{-1}(\Omega)=H_{0}^{1}(\Omega)^{*}$ (see Gasinski and Papageorgiou [6], Theorem 2.4.57, p. 212), we have

$$
\operatorname{div}(a(u) D u) \in H^{-1}(\Omega) .
$$
have

So, if by $\langle\cdot, \cdot\rangle_{0}$ we denote the duality brackets for the pair $\left(H^{-1}(\Omega), H_{0}^{1}(\Omega)\right)$ we

$$
\begin{aligned}
\langle-\operatorname{div}(a(u) D u), h\rangle_{0} & =\int_{\Omega} a(u)(D u, D h)_{\mathbb{R}^{N}} d z \text { for all } h \in H_{0}^{1}(\Omega), \\
\Rightarrow \quad\langle-\operatorname{div}(a(u) D u), h\rangle_{0} & =\int_{\Omega}\left(\vartheta-u-\varphi_{\lambda}^{\prime}(u)\right) h d z \text { for all } h \in H_{0}^{1}(\Omega)(\text { see }(19)), \\
(21 \nRightarrow \quad-\operatorname{div}(a(u(z)) D u(z)) & =\vartheta(z)-u(z)-\varphi_{\lambda}^{\prime}(u(z)) \text { for almost all } z \in \Omega .
\end{aligned}
$$


Then from (19), (20), (21) it follows that

$$
\left\langle a(u) \frac{\partial u}{\partial n}, h\right\rangle_{\partial \Omega}=0 \text { for all } h \in H^{1}(\Omega) .
$$

If by $\gamma_{0}$ we denote the trace map, we recall that

$$
\operatorname{im} \gamma_{0}=H^{\frac{1}{2}, 2}(\partial \Omega)
$$

(see Gasinski and Papageorgiou [6], Theorem 2.4.50, p. 209). Hence from (22) we infer that

$$
\left.\left.\frac{\partial u}{\partial n}\right|_{\partial \Omega}=0 \text { (see hypothesis } H(a)\right) \text {. }
$$

Therefore we have

$$
\left\{\begin{array}{ll}
-\operatorname{div}(a(u)(z) D u(z))+u(z)+\varphi_{\lambda}^{\prime}(u(z))=\vartheta(z) & \text { for almost all } z \in \Omega, \\
\frac{\partial u}{\partial n}=0 & \text { on } \partial \Omega
\end{array}\right\}
$$

From (23) and Proposition 3, we infer that

$$
u \in L^{\infty}(\Omega) .
$$

Then we can use Theorem 2 of Lieberman [12] and conclude that

$$
u \in C^{1}(\bar{\Omega}) .
$$

We establish in what follows the uniqueness of this solution. So, suppose that $v \in C^{1}(\bar{\Omega})$ is another solution. We have

$$
\begin{aligned}
& \hat{a}(u)+u+N_{\varphi_{\lambda}^{\prime}}(u)=\vartheta \text { in } H^{1}(\Omega)^{*}, \\
& \hat{a}(v)+v+N_{\varphi_{\lambda}^{\prime}}(v)=\vartheta \text { in } H^{1}(\Omega)^{*} .
\end{aligned}
$$

Let $k>0$ be the Lipschitz constant in hypothesis $H(a)$. We introduce the following function

$$
\eta_{\epsilon}(s)=\left\{\begin{array}{ll}
\int_{\epsilon}^{s} \frac{d t}{(k t)^{2}} & \text { is } s \geqslant \epsilon \quad \text { with } \epsilon>0 \\
0 & \text { if } s<\epsilon
\end{array}\right. \text {. }
$$

Evidently $\eta_{\epsilon}$ is Lipschitz continuous. So, from Marcus and Mizel [14], we have

$$
\begin{aligned}
& \eta_{\epsilon}(u-v) \in H^{1}(\Omega), \\
& D\left(\eta_{\epsilon}(u-v)\right)=\eta_{\epsilon}^{\prime}(u-v) D(u-v)
\end{aligned}
$$

(see also Gasinski and Papageorgiou [6], Proposition 2.4.25, p. 195). Subtracting (25) from (24), we have

$$
\hat{a}(u)-\hat{a}(v)+(u-v)+\left(N_{\varphi_{\lambda}^{\prime}}(u)-N_{\varphi_{\lambda}^{\prime}(v)}\right)=0 \text { in } H^{1}(\Omega)^{*} .
$$

On $(29)$ we act with $\eta_{\epsilon}(u-v) \in H^{1}(\Omega)$ (see $\left.(27)\right)$. Then

$$
\left\langle\hat{a}(u)-\hat{a}(v), \eta_{\epsilon}(u-v)\right\rangle+\int_{\Omega}(u-v) \eta_{\epsilon}(u-v) d z+\int_{\Omega}\left(\varphi_{\lambda}^{\prime}(u)-\varphi_{\lambda}^{\prime}(v)\right)(u-v) d z=0 .
$$

We have

$$
\int_{\Omega}(u-v) \eta_{\epsilon}(u-v) d z=\int_{\{u-v \geqslant \epsilon\}}(u-v) \eta_{\epsilon}(u-v) d z \geqslant \frac{1}{k} \int_{\{u-v \geqslant \epsilon\}}\left(\frac{u-v}{\epsilon}-1\right) d z(\operatorname{see}(26)) .
$$


Recall that $\varphi_{\lambda}^{\prime}$ is increasing. Therefore

$$
\int_{\Omega}\left(\varphi_{\lambda}^{\prime}(u)-\varphi_{\lambda}^{\prime}(v)\right) \eta_{\epsilon}(u-v) d z=\int_{\{u-v \geqslant \epsilon\}}\left(\varphi_{\lambda}^{\prime}(u)-\varphi_{\lambda}^{\prime}(v)\right) \eta_{\epsilon}(u-v) d z \geqslant 0
$$

(see $(26))$.

We return to (30) and use (31), (32). Then

$$
\begin{aligned}
& \left\langle\hat{a}(u)-\hat{a}(v), \eta_{\epsilon}(u-v)\right\rangle \leqslant 0, \\
\Rightarrow & \int_{\Omega}\left(a(u) D u-a(v) D v, D \eta_{\epsilon}(u-v)\right)_{\mathbb{R}^{N}} d z \leqslant 0, \\
\Rightarrow & 33) \int_{\Omega} a(u)\left(D u-D v, D \eta_{\epsilon}(u-v)\right)_{\mathbb{R}^{N}} d z \leqslant-\int_{\Omega}(a(u)-a(v))\left(D v, D \eta_{\epsilon}(u-v)\right)_{\mathbb{R}^{N}} d z .
\end{aligned}
$$

Let $\Omega_{\epsilon}=\{z \in \Omega:(u-v)(z) \geqslant \epsilon\}$. Then

$$
\begin{aligned}
& \int_{\Omega} a(u)\left(D u-D v, D \eta_{\epsilon}(u-v)\right)_{\mathbb{R}^{N}} d z \\
& =\int_{\Omega_{\epsilon}} a(u) \eta_{\epsilon}^{\prime}(u-v)|D u-D v|^{2} d z(\text { see }(26),(28)) \\
& \left.\geqslant c_{1} \int_{\Omega_{\epsilon}} \frac{|D u-D v|^{2}}{k^{2}(u-v)^{2}} d z \text { (see hypothesis } H(a) \text { and }(26)\right) .
\end{aligned}
$$

Also we have

$$
\begin{aligned}
& -\int_{\Omega}(a(u)-a(v))\left(D v, D \eta_{\epsilon}(u-v)\right)_{\mathbb{R}^{N}} d z \\
& \left.\leqslant \int_{\Omega_{\epsilon}} k(u-v) \eta_{\epsilon}^{\prime}(u-v)(D v, D u-D v)_{\mathbb{R}^{N}} d z \text { (see hypothesis } H(a) \text { and }(28)\right) \\
& =\int_{\Omega_{\epsilon}} \frac{1}{k(u-v)}(D v, D u-D v)_{\mathbb{R}^{N}} d z(\text { see }(26)) \\
(35) \leqslant & \leqslant \mid D v \|_{2}\left(\int_{\Omega_{\epsilon}} \frac{|D u-D v|^{2}}{k^{2}|u-v|^{2}} d z\right)^{1 / 2} \text { (by the Cauchy-Schwarz inequality). }
\end{aligned}
$$

Returning to (33) and using (34), (35) we obtain

$$
\int_{\Omega_{\epsilon}} \frac{|D u-D v|^{2}}{|u-v|^{2}} d z \leqslant \frac{k^{2}}{c_{1}^{2}}\|D v\|_{2}^{2} .
$$

Let $\Omega_{\epsilon}^{*}$ be a connected component of $\hat{\Omega}=\{z \in \Omega ;(u-v)(z)>0\}, \hat{\Omega} \neq \Omega$ (see (31)). We have

$$
\int_{\Omega_{\epsilon}^{*}} \frac{|D u-D v|^{2}}{|u-v|^{2}} d z \leqslant \frac{k^{2}}{c_{1}^{2}}\|D v\|_{2}^{2} \quad \text { with } \Omega_{\epsilon}^{*}=\Omega_{\epsilon} \cap \Omega^{*} .
$$

Consider the function

$$
\gamma_{\epsilon}(y)= \begin{cases}\int_{\epsilon}^{y} \frac{d t}{t} & \text { if } t \geqslant \epsilon \\ 0 & \text { if } t<\epsilon .\end{cases}
$$


This function is Lipschitz continuous and as before from Marcus and Mizel [14], we have

$$
\begin{aligned}
& \gamma_{\epsilon}\left(u(38 y) \in H^{1}(\Omega)\right. \\
& \left.D \gamma_{\epsilon}(39) v\right)=\gamma_{\epsilon}^{\prime}(u-v)(D u-D v)=\frac{1}{u-v}(D u-D v) \text { for almost all } z \in \Omega_{\epsilon}(\text { see }(37)) .
\end{aligned}
$$

Returning to (36) and using (38), (39), we obtain

$$
\int_{\Omega^{*}}\left|D \gamma_{\epsilon}(u-v)\right|^{2} d z \leqslant \frac{k^{2}}{c_{1}^{2}}\|D v\|_{2}^{2} .
$$

Note that $u=v$ on $\partial \Omega^{*}$ (that is, $u-v \in H_{0}^{1}\left(\Omega^{*}\right)$; recall that $u, v \in C^{1}(\bar{\Omega})$ ). Hence

$$
\gamma_{\epsilon}(u-v) \in H_{0}^{1}\left(\Omega^{*}\right)
$$

From (40), (41) and the Poincaré inequality, we have

$$
\int_{\Omega^{*}}\left|\gamma_{\epsilon}(u-v)\right|^{2} d z \leqslant c_{8}\|v\|^{2} \text { for some } c_{8}>0, \text { all } \epsilon>0 \text {. }
$$

If $\left|\Omega^{*}\right|_{N}>0$ (by $|\cdot|_{N}$ we denote the Lebesgue measure on $\mathbb{R}^{N}$ ), then letting $\epsilon \rightarrow 0^{+}$, we reach a contradiction (see $(37)$ ). So, every connected component of the open set

$$
\hat{\Omega}=\{z \in \Omega: u(z)>v(z)\}
$$

is Lebesgue-null. Hence $|\hat{\Omega}|_{N}=0$ and so

$$
u \leqslant v \text {. }
$$

Interchanging the roles of $u, v$ in the above argument, we also obtain

$$
v \leqslant u \text {. }
$$

From (42) and (43) we conclude that

$$
u=v \text {. }
$$

This prove the uniqueness of the solution $u \in C^{1}(\bar{\Omega})$ of the auxiliary problem (16).

Let $C_{n}^{1}(\bar{\Omega})=\left\{u \in C^{1}(\bar{\Omega}):\left.\frac{\partial u}{\partial n}\right|_{\partial \Omega}=0\right\}$ and for every $\lambda>0$ let $K_{\lambda}: L^{\infty}(\Omega) \rightarrow$ $C_{n}^{1}(\bar{\Omega})$ be the map which to each $\vartheta \in L^{\infty}(\Omega)$ assigns the unique solution $u=$ $K_{\lambda}(\vartheta) \in C_{n}^{1}(\bar{\Omega})$ of the auxiliary problem (16) (see Proposition 4 ). The next proposition establishes the continuity properties of this map.

Proposition 5. If hypotheses $H(a), H(\varphi)$ hold then the map $K_{\lambda}: L^{\infty}(\Omega) \rightarrow C_{n}^{1}(\bar{\Omega})$ is sequentially continuous from $L^{\infty}(\Omega)$ furnished with the $w^{*}$-topology into $C_{n}^{1}(\bar{\Omega})$ with the norm topology.

Proof. Suppose that $\vartheta_{n} \stackrel{w^{*}}{\rightarrow} \vartheta$ in $L^{\infty}(\Omega)$ and let $u_{n}=K_{\lambda}\left(\vartheta_{n}\right), u=K_{\lambda}(\vartheta)$.

For every $n \in \mathbb{N}$, we have

$$
\begin{gathered}
\hat{a}\left(u_{n}\right)+u_{n}+N_{\varphi_{\lambda}^{\prime}}\left(u_{n}\right)=\vartheta_{n} \\
\Rightarrow \quad-\operatorname{div}\left(a\left(u_{n}(z)\right) D u_{n}(z)\right)+u_{n}(z)+\varphi_{\lambda}^{\prime}\left(u_{n}(z)\right)=\vartheta_{n}(z) \\
\text { for almost all } z \in \Omega, \frac{\partial u_{n}}{\partial n}=0 \text { on } \partial \Omega .
\end{gathered}
$$


On (44) we act with $u_{n} \in C_{n}^{1}(\bar{\Omega})$. Then

$$
\begin{aligned}
& \int_{\Omega} a\left(u_{n}\right)\left|D u_{n}\right|^{2} d z+\left\|u_{n}\right\|_{2}^{2}+\int_{\Omega} \varphi_{\lambda}^{\prime}\left(u_{n}\right) u_{n} d z=\int_{\Omega} \vartheta_{n} u_{n} d z \\
\Rightarrow \quad & c_{1}\left\|D u_{n}\right\|_{2}^{2}+\left\|u_{n}\right\|_{2}^{2} \leqslant c_{9}\left\|u_{n}\right\| \text { for some } c_{9}>0, \text { all } n \in \mathbb{N} \\
& \text { (see hypothesis } \left.H(a) \text { and recall that } \varphi_{\lambda}^{\prime} \text { is increasing with } \varphi_{\lambda}^{\prime}(0)=0\right) \\
\Rightarrow \quad & \left\|u_{n}\right\| \leqslant c_{10} \text { for some } c_{10}>0, \text { all } n \in \mathbb{N}, \\
\Rightarrow \quad & \left\{u_{n}\right\}_{n \geqslant 1} \subseteq H^{1}(\Omega) \text { is bounded. }
\end{aligned}
$$

By passing to a subsequence if necessary, we may assume that

$$
u_{n} \stackrel{w}{\rightarrow} \hat{u} \text { in } H^{1}(\Omega) \text { and } u_{n} \rightarrow \hat{u} \text { in } L^{2}(\Omega) .
$$

Then for every $h \in H^{1}(\Omega)$ we have

$$
\left\langle\hat{a}\left(u_{n}\right), h\right\rangle=\int_{\Omega} a\left(u_{n}\right)\left(D u_{n}, D h\right)_{\mathbb{R}^{N}} d z \rightarrow \int_{\Omega} a(\hat{u})(D \hat{u}, D h)_{\mathbb{R}^{N}} d z=\langle\hat{a}(\hat{u}), h\rangle
$$

(see (46) and hypothesis $H(a))$,

$\left(47 \nRightarrow \hat{a}\left(u_{n}\right) \stackrel{w}{\rightarrow} \hat{a}(\hat{u})\right.$ in $H^{1}(\Omega)^{*}$.

Therefore, if in (44) we pass to the limit as $n \rightarrow \infty$ and use (46), (47), then

$$
\begin{aligned}
& \hat{a}(\hat{u})+\hat{u}+N_{\varphi_{\lambda}^{\prime}}(\hat{u})=\vartheta, \\
\Rightarrow \quad & \hat{u}=u \in C^{1}(\bar{\Omega})=\text { the unique solution of (16) (see Proposition 4). }
\end{aligned}
$$

From (45) and Proposition 3, (recall that $\left\{u_{n}\right\}_{n \geqslant 1} \subseteq H^{1}(\Omega)$ is bounded), we see that we can find $c_{11}>0$ such that

$$
\left\|u_{n}\right\|_{\infty} \leqslant c_{11} \text { for all } n \in \mathbb{N} \text {. }
$$

Then (48) and Theorem 2 of Lieberman [12] imply that we can find $\alpha \in(0,1)$ and $c_{12}>0$ such that

$$
u_{n} \in C^{1, \alpha}(\bar{\Omega}),\left\|u_{n}\right\|_{C^{1, \alpha}(\bar{\Omega})} \leqslant c_{12} \text { for all } n \in \mathbb{N} .
$$

From (49), the compact embedding of $C^{1, \alpha}(\bar{\Omega})$ into $C^{1}(\bar{\Omega})$ and $(46)$, we have

$$
\begin{aligned}
& u_{n} \rightarrow u \text { in } C^{1}(\bar{\Omega}), \\
\Rightarrow \quad & K_{\lambda}\left(\vartheta_{n}\right) \rightarrow K_{\lambda}(\vartheta) \text { in } C^{1}(\bar{\Omega}) .
\end{aligned}
$$

This proves that $K_{\lambda}$ is sequentially continuous from $L^{\infty}(\Omega)$ with the $w^{*}$-topology into $C_{n}^{1}(\bar{\Omega})$ with the norm topology.

We consider the following approximation to problem (1):

$\left(50\left\{\begin{array}{ll}\operatorname{div}(a(u(z)) D u(z)) \in \varphi_{\lambda}^{\prime}(u(z))+F(z, u(z), D u(z)) & \text { in } \Omega, \\ \frac{\partial u}{\partial n}=0 & \text { on } \partial \Omega, \lambda>0 .\end{array}\right\}\right.$

Proposition 6. If hypotheses $H(a), H(\varphi), H(F)$ hold and $\lambda>0$, then problem (50) admits a solution $u_{\lambda} \in C^{1}(\bar{\Omega})$.

Proof. Consider the multifunction $N: C_{n}^{1}(\bar{\Omega}) \rightarrow 2^{L^{\infty}(\Omega)}$ defined by

$$
N(u)=\left\{f \in L^{\infty}(\Omega): f(z) \in F(z, u(z), D u(z)) \text { for almost all } z \in \Omega\right\} .
$$

Hypotheses $H(F)(i),(i i)$ imply that the multifunction $z \mapsto F(z, u(z), D u(z))$ admits a measurable selection (see $\mathrm{Hu}$ and Papageorgiou [10, p. 21]) and then hypothesis $H(F)($ iii $)$ implies that this measurable selection belongs in $L^{\infty}(\Omega)$ and so 
$N(\cdot)$ has nonempty values, which is easy to see that they are $w^{*}$-compact (Alaoglu's theorem) and convex. Let

$$
N_{1}(u)=u-N(u) \text { for all } u \in C_{n}^{1}(\bar{\Omega}) .
$$

We consider the following fixed point problem

$$
u \in K_{\lambda} N_{1}(u) .
$$

Let $E=\left\{u \in C_{n}^{1}(\bar{\Omega}): u \in t K_{\lambda} N_{1}(u)\right.$ for some $\left.t \in(0,1)\right\}$.

Claim 2. The set $E \subseteq C_{n}^{1}(\bar{\Omega})$ is bounded.

Let $u \in E$. Then from the definitions of $K_{\lambda}$ and $N_{1}$ we have

$$
\hat{a}\left(\frac{1}{t} u\right)+\frac{1}{t} u+N_{\varphi_{\lambda}^{\prime}}\left(\frac{1}{t} u\right)=u-f \text { with } f \in N(u) .
$$

On (52) we act with $u \in H^{1}(\Omega)$. Using hypothesis $H(a)$, we obtain

$$
\frac{c_{1}}{t}\|D u\|_{2}^{2}+\frac{1}{t}\|u\|_{2}^{2} \leqslant\|u\|_{2}^{2}-\int_{\Omega} f u d z
$$

(recall that $\varphi_{\lambda}^{\prime}$ is increasing and $\varphi_{\lambda}^{\prime}(0)=0$ ),

$(53) \Rightarrow \quad c_{1}\|D u\|_{2}^{2} \leqslant(t-1)\|u\|_{2}^{2}-t \int_{\Omega} f u d z \leqslant-t \int_{\Omega} f u d z$ (recall that $\left.t \in(0,1)\right)$.

Hypothesis $H(F)(v)$ implies that

$$
-t \int_{\Omega} f u d z \leqslant t c_{3}\|u\|_{2}^{2}+t c_{4} \int_{\Omega}|u|^{2}|D u| d z+\int_{\Omega} \gamma_{3}(z)|u|^{2} d z .
$$

Let $M>0$ be as postulated by hypothesis $H(F)(i v)$. We will show that

$$
\|u\|_{\infty} \leqslant M \text {. }
$$

To this end let $\hat{\sigma}_{0}(z)=|u(z)|^{2}$. Let $z_{0} \in \bar{\Omega}$ be such that

$$
\hat{\sigma}_{0}\left(z_{0}\right)=\max _{\bar{\Omega}} \hat{\sigma}_{0} \text { (recall that } u \in E \subseteq C_{n}^{1}(\bar{\Omega}) \text { ). }
$$

Suppose that $\hat{\sigma}_{0}\left(z_{0}\right)>M^{2}$. First assume that $z_{0} \in \Omega$. Then

$$
\begin{aligned}
& 0=D \hat{\sigma}_{0}\left(z_{0}\right)=2 u\left(z_{0}\right) D u\left(z_{0}\right), \\
\Rightarrow \quad & D u\left(z_{0}\right)=0\left(\text { since }\left|u\left(z_{0}\right)\right|>M\right) .
\end{aligned}
$$

Let $\delta, \eta>0$ be as in hypothesis $H(F)(i v)$. Since $\hat{\sigma}_{0}\left(z_{0}\right)>M^{2}$ and $u \in C_{n}^{1}(\bar{\Omega})$ we can find $\delta_{1}>0$ such that

$$
\begin{aligned}
z \in \bar{B}_{\delta_{1}}\left(z_{0}\right)=\{z \in \Omega: \mid & \left.\left|z-z_{0}\right| \leqslant \delta_{1}\right\} \Rightarrow\left|u(z)-u\left(z_{0}\right)\right|+|D u(z)| \leqslant \delta \\
& \left(\text { recall that } D u\left(z_{0}\right)=0\right), \\
(55) \Rightarrow t f(z) u(z)+t c_{1}|D u(z)|^{2} \geqslant & t \eta>0 \text { for almost all } z \in \bar{B}_{\delta_{1}}\left(z_{0}\right) \\
& \quad(\text { see hypothesis } H(F)(i v)) .
\end{aligned}
$$

From (52) as before (see the proof of Proposition 4), we have (56)

$-\operatorname{div}\left(a\left(\frac{1}{t} u(z)\right) D\left(\frac{1}{t} u\right)(z)\right)+\varphi_{\lambda}^{\prime}\left(\frac{1}{t} u(z)\right)=\left(1-\frac{1}{t}\right) u(z)-f(z)$ for almost all $z \in \Omega$. 
Using (56) in (55), we obtain

$$
\left[\operatorname{div}\left(a\left(\frac{1}{t} u(z)\right) D u(z)\right)-t \varphi_{\lambda}^{\prime}\left(\frac{1}{t} u(z)\right)+(t-1) u(z)\right] u(z)+t c_{1}|D u(z)|^{2} \geqslant t \eta
$$

for almost all $z \in \bar{B}_{\delta_{1}}\left(z_{0}\right)$.

We integrate over $\bar{B}_{\delta_{1}}\left(z_{0}\right)$ and use the fact that $t \in(0,1)$. Then

$$
\begin{gathered}
\int_{\bar{B}_{\delta_{1}}\left(z_{0}\right)} \operatorname{div}\left(a\left(\frac{1}{t} u\right) D u\right) u d z-t \int_{\bar{B}_{\delta_{1}}\left(z_{0}\right)} \varphi_{\lambda}^{\prime}\left(\frac{1}{t} u\right) u d z+t c_{1} \int_{\bar{B}_{\delta_{1}}\left(z_{0}\right)}|D u|^{2} d z \geqslant \mu \eta\left|\bar{B}_{\delta_{1}}\left(z_{0}\right)\right|_{N} \\
\Rightarrow \int_{\bar{B}_{\delta_{1}}\left(z_{0}\right)} \operatorname{div}\left(a\left(\frac{1}{t} u\right) D u\right) u d z+t c_{1} \int_{\bar{B}_{\delta_{1}}\left(z_{0}\right)}|D u|^{2} d z>0 \\
\text { (recall that } \left.\varphi_{\lambda}^{\prime} \text { is increasing and } \varphi_{\lambda}^{\prime}(0)=0\right) .
\end{gathered}
$$

Using the nonlinear Green's identity (see Gasinski and Papageorgiou [6], Theorem 2.4 .53 , p. 210), we obtain

$$
0<-\int_{\bar{B}_{\delta_{1}}\left(z_{0}\right)} a\left(\frac{1}{t} u\right)|D u|^{2} d z+\int_{\partial \bar{B}_{\delta_{1}}\left(z_{0}\right)} a\left(\frac{1}{t} u\right) \frac{\partial u}{\partial n} u d \sigma+t c_{1} \int_{\bar{B}_{\delta_{1}}\left(z_{0}\right)}|D u|^{2} d z
$$

Here by $\sigma(\cdot)$ we denote the $(N-1)$-dimensional Hausdorff (surface) measure defined on $\partial \Omega$. Hence we ahve

$$
\begin{aligned}
0<-c_{1} \int_{\bar{B}_{\delta_{1}}\left(z_{0}\right)}|D u|^{2} d z+\int_{\partial \bar{B}_{\delta_{1}}\left(z_{0}\right)} a\left(\frac{1}{t} u\right) \frac{\partial u}{\partial n} u d \sigma+t c_{1} \int_{\bar{B}_{\delta_{1}}\left(z_{0}\right)}|D u|^{2} d z \\
\quad \text { (see hypothesis } H(a)), \\
\left.\Rightarrow \quad 0<\int_{\partial \bar{B}_{\delta_{1}}\left(z_{0}\right)} a\left(\frac{1}{t} u\right) \frac{\partial u}{\partial n} u d \sigma \text { (recall that } t \in(0,1)\right), \\
\left.\Rightarrow \quad 0<c_{2} \int_{\partial \bar{B}_{\delta_{1}}\left(z_{0}\right)} \frac{\partial u}{\partial n} u d \sigma \text { (see hypothesis } H(a)\right) .
\end{aligned}
$$

Thus we can find a continuous path $\{c(t)\}_{t \in[0,1]}$ in $\bar{B}_{\delta_{1}}\left(z_{0}\right)$ with $c(0)=z_{0}$ such that

$$
\begin{aligned}
& a<\int_{0}^{1} u(c(t))\left(D u(c(t)), c^{\prime}(t)\right)_{\mathbb{R}^{N}} d t \\
& =\int_{0}^{1} \frac{1}{2} \frac{d}{d t} u(c(t))^{2} d t \\
& =\frac{1}{2}\left[u(c(1))-u\left(z_{0}\right)\right], \\
\Rightarrow \quad & u\left(z_{0}\right)<u(c(1)),
\end{aligned}
$$

which contradicts the choice of $z_{0}$. So, we cannot have $z_{0} \in \Omega$.

Therefore we assume that $z_{0} \in \partial \Omega$. Since $u \in C_{n}^{1}(\bar{\Omega})$, again we have $D u\left(z_{0}\right)=0$ and so the above argument applies with $\partial \bar{B}_{\delta_{1}}\left(z_{0}\right)$ replaced by $\partial \bar{B}_{\delta_{1}}\left(z_{0}\right) \cap \Omega$.

Hence we have proved that

(58) $\|u\|_{\infty} \leqslant M$ for all $u \in E$ (here $M>0$ is as in hypothesis $H(F)(i v)$ ). 
We use (58) in (54) and have

$$
\begin{aligned}
& -t \int_{\Omega} f u d z \leqslant t c_{13}\left(1+\|D u\|_{2}\right) \text { for some } c_{13}>0 \\
\Rightarrow & c_{1}\|D u\|_{2}^{2} \leqslant c_{13}\left(1+\|D u\|_{2}\right)(\text { see }(53) \text { and recall } t \in(0,1)), \\
\Rightarrow & \|D u\|_{2} \leqslant c_{14} \text { for some } c_{14}>0, \text { all } u \in E .
\end{aligned}
$$

Then (58), (59) imply that $E \subseteq H^{1}(\Omega)$ is bounded. Invoking Theorem 2 of Lieberman [12], we can find $c_{15}>0$ such that

$$
\begin{aligned}
& \|u\|_{C^{1}(\bar{\Omega})} \leqslant c_{15} \text { for all } u \in E, \\
\Rightarrow \quad & E \subseteq C_{n}^{1}(\bar{\Omega}) \text { is bounded. }
\end{aligned}
$$

This proves Claim 2.

Recall that hypotheses $H(F)(i),(i i),(i i i)$ imply that $N_{1}$ is a multifunction which is usc from $C_{n}^{1}(\bar{\Omega})$ with the norm topology into $L^{\infty}(\Omega)$ with the $w^{*}$-topology (see $\mathrm{Hu}$ and Papageorgiou [10, p. 21]). This fact, Proposition 5 and Claim 2, permit the use of Theorem 1. So, we can find $u_{\lambda} \in C_{n}^{1}(\bar{\Omega})$ such that

$$
\begin{aligned}
& u_{\lambda} \in K_{\lambda} N_{1}\left(u_{\lambda}\right), \\
\Rightarrow \quad & u_{\lambda} \in C_{n}^{1}(\bar{\Omega}) \text { is a solution of problem (50). }
\end{aligned}
$$

Now we are ready for the existence theorem concerning problem (1).

Theorem 7. If hypotheses $H(a), H(\varphi), H(F)$ hold, then problem (1) admits a solution $u \in C_{n}^{1}(\bar{\Omega})$.

Proof. Let $\lambda_{n} \rightarrow 0^{+}$. From Proposition 6, we know that problem (50) (with $\lambda=\lambda_{n}$ ) has a solution $u_{n}=u_{\lambda_{n}} \in C_{n}^{1}(\bar{\Omega})$. Moreover, from the proof of that proposition, we have

$$
\left\|u_{n}\right\|_{\infty} \leqslant M \text { for all } n \in \mathbb{N} \text { (see (58)). }
$$

For every $n \in \mathbb{N}$, we have

(61) $\hat{a}\left(u_{n}\right)+N_{\varphi_{\lambda_{n}}^{\prime}}\left(u_{n}\right)+f_{n}=0$ with $f_{n} \in N\left(u_{n}\right)$ (see the proof of Proposition 6).

On (61) we act with $u_{n}$ and obtain

$$
\begin{aligned}
& c_{1}\left\|D u_{n}\right\|_{2}^{2} \leqslant\left\|f_{n}\right\|_{2}\left\|u_{n}\right\|_{2} \\
& \left(\text { see hypothesis } H(a) \text { and recall that } \varphi_{\lambda}^{\prime}(s) s \geqslant 0 \text { for all } s \in \mathbb{R}\right), \\
\Rightarrow \quad & \left\|D u_{n}\right\|_{2} \leqslant c_{16} \text { for some } c_{16}>0, \text { all } n \in \mathbb{N} \\
& \text { (see (60) and hypothesis } H(F)(\text { iii })) .
\end{aligned}
$$

From (60) and (62) it follows that

$$
\left\{u_{n}\right\}_{n \geqslant 1} \subseteq H^{1}(\Omega) \text { is bounded. }
$$

So, by passing to a subsequence if necessary, we may assume that

$$
u_{n} \stackrel{w}{\rightarrow} u \text { in } H^{1}(\Omega) \text { and } u_{n} \rightarrow u \text { in } L^{2}(\Omega) .
$$

Acting on (61) with $N_{\varphi_{\lambda_{n}}^{\prime}}\left(u_{n}\right)(\cdot)=\varphi_{\lambda_{n}}^{\prime}\left(u_{n}(\cdot)\right) \in C(\bar{\Omega}) \cap H^{1}(\Omega)$ (recall that $\varphi_{\lambda_{n}}^{\prime}(\cdot)$ is Lipschitz continuous and see Marcus and Mizel [14]), we have

$$
\int_{\Omega} a\left(u_{n}\right)\left(D u_{n}, D \varphi_{\lambda}^{\prime}\left(u_{n}\right)\right)_{\mathbb{R}^{N}} d z+\left\|N_{\varphi_{\lambda_{n}}^{\prime}}\left(u_{n}\right)\right\|_{2}^{2}=-\int_{\Omega} f_{n} \varphi_{\lambda_{n}}^{\prime}\left(u_{n}\right) d z .
$$


From the chain rule of Marcus and Mizel [14], we have

$$
D \varphi_{\lambda_{n}}^{\prime}\left(u_{n}\right)=\varphi_{\lambda_{n}}^{\prime \prime}\left(u_{n}\right) D u_{n} .
$$

Since $\varphi_{\lambda_{n}}^{\prime}(\cdot)$ is increasing (recall that $\varphi_{\lambda_{n}}$ is convex), we have

$$
\varphi_{\lambda_{n}}^{\prime \prime}\left(u_{n}(z)\right) \geqslant 0 \text { for almost all } z \in \Omega \text {. }
$$

Using (64), (65) and hypothesis $H(a)$, we see that

$$
0 \leqslant \int_{\Omega} a\left(u_{n}\right)\left(D u_{n}, D \varphi_{\lambda_{n}}^{\prime}\left(u_{n}\right)\right)_{\mathbb{R}^{N}} d z
$$

Using (66) in (63), we obtain

$$
\begin{aligned}
&\left\|N_{\varphi_{\lambda_{n}}^{\prime}}\left(u_{n}\right)\right\|_{2}^{2} \leqslant\left\|f_{n}\right\|_{2}\left\|N_{\varphi_{\lambda_{n}}^{\prime}}\left(u_{n}\right)\right\|_{2} \text { for all } n \in \mathbb{N}, \\
& \Rightarrow \quad\left\|N_{\varphi_{\lambda_{n}}^{\prime}}\left(u_{n}\right)\right\|_{2} \leqslant\left\|f_{n}\right\|_{2} \leqslant c_{17} \text { for some } c_{17}>0, \text { all } n \in \mathbb{N} \\
&(\text { see }(60) \text { and hypothesis } \mathrm{H}(\mathrm{F})(\text { iii })) \\
& \Rightarrow \quad\left\{N_{\varphi_{\lambda_{n}}^{\prime}}\left(u_{n}\right)\right\}_{n \geqslant 1} \subseteq L^{2}(\Omega) \text { is bounded. }
\end{aligned}
$$

So, we may assume that

$$
N_{\varphi_{\lambda_{n}}^{\prime}} \stackrel{w}{\rightarrow} g \text { and } f_{n} \stackrel{w}{\rightarrow} f \text { in } L^{2}(\Omega) .
$$

As in the proof of Proposition 5 (see (47)), we show that

$$
\hat{a}\left(u_{n}\right) \stackrel{w}{\rightarrow} \hat{a}(u) \text { in } H^{1}(\Omega)^{*} .
$$

So, if in (61) we pass to the limit as $n \rightarrow \infty$ and use (67) and (68), we obtain $\hat{a}(u)+g+f=0$,

(69) $-\operatorname{div}(a(u(z)) D u(z))+g(z)+f(z)=0$ for almost all $z \in \Omega, \frac{\partial u}{\partial n}=0$ on $\partial \Omega$ (see the proof of Proposition 4).

Because of (60) and Theorem 2 of Lieberman [12], we know that there exist $\alpha \in(0,1)$ and $c_{18}>0$ such that

$$
u_{n} \in C^{1, \alpha}(\bar{\Omega}),\left\|u_{n}\right\|_{C^{1, \alpha}(\bar{\Omega})} \leqslant c_{18} \text { for all } n \in \mathbb{N},
$$

(7@ $u_{n} \rightarrow u$ in $C^{1}(\bar{\Omega})$ (recall that $C^{1, \alpha}(\bar{\Omega})$ is embedded compactly into $C^{1}(\bar{\Omega})$ ).

Recall that

$$
f_{n}(z) \in F\left(z, u_{n}(z), D u_{n}(z)\right) \text { for almost all } z \in \Omega \text {, all } n \in \mathbb{N} \text {, }
$$$$
\Rightarrow \quad f(z) \in F(z, u(z), D u(z))
$$

(see (67), (70), hypothesis H(F)(ii) and Proposition 6.6.33, p. 521 of [19]),

(71) $f \in N(u)$.

Also, from (67), (70) and Corollary 3.2.51, p. 179 of [19], we have

$$
g(z) \in \partial \varphi(u(z)) \text { for almost all } z \in \Omega .
$$
(1).

So, from (69), (71), (72) we conclude that $u \in C_{n}^{1}(\bar{\Omega})$ is a solution of problem 


\section{EXAMPLES}

In this section we present two concrete situations illustrating our result.

For the first, let $\mu \leqslant 0$ and consider the function

$$
\varphi(x)= \begin{cases}+\infty & \text { if } x<\mu \\ 0 & \text { if } \mu \leqslant x\end{cases}
$$

Evidently we have

$$
\varphi \in \Gamma_{0}(\mathbb{R}) \text { and } 0 \in \partial \varphi(0) .
$$

In fact note that

$$
\partial \varphi(x)= \begin{cases}\emptyset & \text { if } x<\mu \\ \mathbb{R}_{-} & \text {if } x=\mu \\ \{0\} & \text { if } \mu<x\end{cases}
$$

Also consider a Carathéodory function $f: \Omega \times \mathbb{R} \times \mathbb{R}^{N} \rightarrow \mathbb{R}$ which satisfies hypotheses $H(F)(i i i),(i v),(v)$. For example, we can have the following function (for the sake of simplicity we drop the $z$-dependence):

$$
f(x, \xi)=c \sin x+x-\ln (1+|\xi|)+\vartheta \text { with } c_{1} \vartheta>0 .
$$

Then according to Theorem 7 , we can find a solution $u_{0} \in C^{1}(\bar{\Omega})$ for the following problem:

$$
\left\{\begin{array}{l}
\operatorname{div}(a(u(z)) D u(z)) \leqslant f(z, u(z), D u(z)) \text { for almost all } z \in\{u=\mu\}, \\
\operatorname{div}(a(u(z)) D u(z))=f(z, u(z), D u(z)) \text { for almost all } z \in\{\mu<u\}, \\
u(z) \geqslant \mu \text { for all } z \in \bar{\Omega}, \frac{\partial u}{\partial n}=0 \text { on } \partial \Omega .
\end{array}\right\}
$$

For the second example, we consider a variational-hemivariational inequality. Such problems arise in mechanics, see Panagiotopoulos [18]. So, let $j(z, x)$ be a locally Lipschitz integrand (that is, for all $x \in \mathbb{R}, z \mapsto j(z, x)$ is measurable and for almost all $z \in \Omega, x \mapsto j(z, x)$ is locally Lipschitz). By $\partial_{c} j(z, x)$ we denote the Clarke subdifferential of $j(z, \cdot)$. We impose the following conditions on the integrand $j(z, x)$ :

(a) for almost all $z \in \Omega$, all $x \in \mathbb{R}$ and all $v \in \partial j(z, x)$

$$
|v| \leqslant \hat{c}_{1}(1+|x|) \text { for almost all } z \in \Omega \text {, all } x \in \mathbb{R} \text {, with } \hat{c}_{1}>0 ;
$$

(b) $0<\hat{c}_{2} \leqslant \liminf _{x \rightarrow \pm \infty} \frac{v}{x} \leqslant \limsup _{x \rightarrow \pm \infty} \frac{v}{x} \leqslant \hat{c}_{3}$ uniformly for almost all $z \in \Omega$, all $v \in \partial j(z, x)$

(c) $-\hat{c}_{4} \leqslant \liminf _{x \rightarrow 0} \frac{v}{x} \leqslant \limsup _{x \rightarrow 0} \frac{v}{x} \leqslant \hat{c}_{5}$ uniformly for almost all $z \in \Omega$, all $v \in \partial j(z, x)$ and with $\hat{c}_{4}, \hat{c}_{5}>0$.

A possible choice of $j$ is the following (as before for the sake of simplicity we drop the $z$-dependence):

$$
j(x)=\left\{\begin{array}{ll}
\frac{1}{p}|x|^{p}-\cos \left(\frac{\pi}{2}|x|\right) & \text { if }|x| \leqslant 1 \\
\frac{1}{2} x^{2}-\ln |x|+c & \text { if } 1<|x|
\end{array} \quad \text { with } c=\frac{1}{p}-\frac{1}{2}, 1<p .\right.
$$

We set

$$
F(z, x, \xi)=\partial j(z, x)+x|\xi|+\vartheta(z) \text { with } \vartheta \in L^{\infty}(\Omega) .
$$

Using (a),(b),(c) above, we can see that hypotheses $H(F)$ are satisfied. 
Also, suppose that $\varphi$ satisfies hypothesis $H(\varphi)$. Two specific choices of interest are

$$
\varphi(x)=|x| \text { and } \varphi(x)=i_{[-1,1]}(x)= \begin{cases}0 & \text { if }|x| \leqslant 1 \\ +\infty & \text { if } 1<|x|\end{cases}
$$

Then the following problem admits a solution $u_{0} \in C^{1}(\bar{\Omega})$ :

$$
\left\{\begin{array}{ll}
\operatorname{div}(a(u(z)) D u(z)) \in \partial \varphi(u(z))+F(z, u(z), D u(z)) & \text { in } \Omega, \\
\frac{\partial u}{\partial n}=0 & \text { on } \partial \Omega .
\end{array}\right\}
$$

The case $\varphi \equiv 0$ (hemivariational inequalities) incorporates problems with discontinuities in which we fill-in the gaps at the jump discontinuities.

Acknowledgments. This research was supported by the Slovenian Research Agency grants P1-0292, J1-7025, J1-6721, and J1-5435, and the Romanian National Authority for Scientific Research and Innovation CNCS-UEFISCDI, grant PN-IIPT-PCCA-2013-4-0614.

\section{REFERENCES}

[1] H. Amann, M. Crandall, On some existence theorems for semi-linear elliptic equations, Indiana Univ. Math. J. 27 (1978), 779-790.

[2] D. Arcoya, J. Carmona, P.J. Martinez Aparicio, Elliptic obstacle problems with natural growth on the gradient and singular nonlinear terms, Advanced Nonlin. Studies 7 (2007), 299-318.

[3] R. Bader, A topological fixed-point index theory for evolution inclusions, Z. Anal. Anwend. 20 (2001), 3-15.

[4] F. Clarke, Optimization and Nonsmooth Analysis, Wiley-Interscience, New York, 1983.

[5] D. de Figueiredo, M. Girardi, M. Matzeu, Semilinear singular elliptic equations with dependence on the gradient via mountain pass techniques, Differential Integral Equations $\mathbf{1 7}$ (2004), 119-126.

[6] L. Gasinski, N.S. Papageorgiou, Nonlinear Analysis, Chapman \& Hall/CRC, Boca Raton, FL, 2006.

[7] L. Gasinski, N.S. Papageorgiou, Exercises in Analysis: Part 1, Springer, New York, 2014.

[8] M. Girardi, M. Matzeu, Positive and negative solutions of a quasilinear elliptic equation by a mountain pass method and truncature techniques, Nonlinear Anal. 59 (2004), 199-210.

[9] P. Hartman, Ordinary Differential Equations, Wiley, New York, 1964.

[10] S. Hu, N.S. Papageorgiou, Handbook of Multivalued Analysis. Part II: Applications, Kluwer, Dordrecht, The Netherlands, 2000.

[11] W. Knobloch, On the existence of periodic solutions for second order vector differential equations, J. Differential Equations 9 (1971), 67-85.

[12] G. Lieberman, Boundary regularity for solutions of degenerate elliptic equations, Nonlinear Anal. 12 (1988), 1203-1219.

[13] N.H. Loc, K. Schmitt, Bernstein-Nagumo conditions and solutions to nonlinear differential inequalities, Nonlinear Anal. 75 (2012), 4664-4671.

[14] M. Marcus, V. Mizel, Absolute continuity on tracks and mappings of Sobolev spaces, Arch. Rational Mech. Anal. 45 (1972), 294-320.

[15] M. Matzeu, R. Servadei, Semilinear elliptic variational inequalities with dependence on the gradient via mountain pass techniques, Nonlinear Anal. 72 (2010), 4347-4359.

[16] J. Mawhin, Boundary value problems for nonlinear second order differential equations, $J$. Differential Equations 16 (1974), 257-269.

[17] A. Mokrane, F. Murat, The Lewy-Stampacchia inequality for the obstacle problem with quadratic growth in the gradient, Annali Mat. Pura. Appl. 184 (2005), 347-360.

[18] P.D. Panagiotopoulos, Hemivariational Inequalities and Applications in Mechanics and Engineering, Springer-Verlag, New York, 1993.

[19] N.S. Papageorgiou, S. Kyritsi, Handbook of Applied Analysis, Springer, New York (2009).

[20] S.I. Pohozaev, Equations of the type $\Delta u=f(x, u, D u)$ (Russian), Mat. Sb. (NS) 113 (155) (1980), 321-338. 
(N.S. Papageorgiou) National Technical University, Department of Mathematics, Zografou Campus, 15780 Athens, Greece \& Institute of Mathematics, Physics and Mechanics, 1000 Ljubluana, Slovenia

E-mail address: npapg@math.ntua.gr

(V.D. Rădulescu) Institute of Mathematics, Physics and Mechanics, 1000 Luubljana, Slovenia \& Faculty of Applied Mathematics, AGH University of Science and Technology, 30-059 Kraków, Poland

E-mail address: vicentiu.radulescu@imfm.si

(D.D. Repovš) Faculty of Education and Faculty of Mathematics and Physics, UniverSity of Luubluana, \& Institute of Mathematics, Physics and Mechanics, 1000 Luubluana, SLOVENIA

E-mail address: dusan.repovs@guest.arnes.si 